\title{
Tax Instruments and the Achievement of Tax Equity
}

\author{
A. Vernizzi and S. Pellegrino
}

\begin{abstract}
Deductions and tax credits are instruments usually adopted by tax systems, in addition to marginal tax rates, to get the desired tax revenue, together with other fiscal policy objectives, respecting vertical and horizontal equity requirements. The effect of marginal tax rates, deductions and tax credits is here decomposed in order to detect whether and to which extent each of these three instruments violate tax equity.
\end{abstract}

Keywords - Equity in personal income tax, Equity violation decomposition, Kakwani-Lambert axioms.

\section{INTRODUCTION}

The effects on taxes, due to marginal tax rates, deductions and tax credit, were first analyzed by Pfäler [7]; then the analysis was refined by Lambert [3] and, recently by Onrubia et Al. [5]. The Pfäler-Lambert-Onrubia et al. approach only focus on how the redistributive effect is achieved. This paper explores which features of the tax contribute the most to the removal of a real-world tax from a perfectly equal one. Kakwani and Lambert [2] (henceforth KL) state that tax should increase monotonically with respect to people's ability to pay (Axiom 1); richer people should pay taxes at higher rates (Axiom 2); no re-ranking should occur in people's living standards (Axiom 3). A violation of Axiom 1 automatically entails a violation of Axiom 2, although not necessarily the other way round. Moreover, Axiom 3 can be violated only if Axiom 2 (and consequently Axiom 1) holds. For the sake of simplicity, we limit our analysis to Kakwani and Lambert (henceforth KL) axiom 1 and axiom 3. Our approach is inspired by the works by Podder [8] [9] and Podder and Chatterjee [10]. The goal is to measure the share of the redistributive effect due to each element of the tax, given the application of all the other tax elements, that is to say from an ex-post overall perspective.

\section{Preliminary Definitions}

Let $X$ be the distribution of gross (pre-tax) incomes order in non decreasing order. Let $D$ be the distribution of deduction ordered in non decreasing order and $D_{\mid X}$ the distribution of deduction ranked by $X$. Analogously we define $C$ and $C_{\mid X}$ the distribution of tax-credits. Let $V$ (and $V_{\mid X}$ ) the distribution which is given by the application of the tax schedule to $X$, Let $S$ (and $S_{\mid X}$ ) the distribution which is given after having applied the tax schedule to $B=X-D$. And finally let's define by $Y$ (and $Y_{\mid X}$ ) the distribution of net incomes, i.e. $Y_{\mid X}=X-S_{\mid X}+C_{\mid X}=X-T_{\mid X}$; $T\left(T_{\mid X}\right)$ are net taxes. $\tilde{T}\left(\tilde{T}_{\mid X}\right)$ are average net tax-rates.

A. Vernizzi, DEMM, Università degli Studi di Milano, Italy. S. Pellegrino, ESOMAS, Università degli Studi di Torino, Italy.
The redistributive effect $R E$, is defined as

$R E=G_{X}-G_{Y}=G_{X}-C_{Y \mid X}-R_{Y \mid X}=\tau K-R_{Y \mid X}$.

In (1) $\tau$ is the ratio between the tax average and the average of the after tax income, i.e. $\tau=\mu_{T} / \mu_{Y} ; C_{Y \mid X}$ is the pseudo Gini (concentration) coefficient of $Y_{\mid X}$ (i.e. $Y$ ranked by $X$ ).

$R_{Y}=\left(G_{Y}-C_{Y \mid X}\right)$ is the Atkinson-Plotnick-Kakwani re-ranking index; $K$ is the Kakwany progressivity index, $K=\left(C_{T \mid X}-G_{X}\right)$ We recall the definition of the Reynolds-Smolensky index $R S=G_{X}-C_{Y \mid X}=\tau K$,

from which $R E=R S-R_{Y \mid X}$.

According to $\mathrm{KL} A V_{1}^{T}=\tau\left(G_{T}-C_{T \mid X}\right)$ measures the violation of axiom 1, as it reveals whether taxes are not ranked as gross incomes as they should. $A V_{2}^{T}=\tau\left(G_{\tilde{T}}-C_{\tilde{T} \mid X}\right)$ measures the violation of axiom 2 , as it reveals whether average net tax-rates are not ranked as gross incomes as they should. $A V_{3}^{T}=G_{Y}-C_{Y \mid X}$ measures the violation of axiom 3 , as it reveals whether after tax incomes are not ranked as gross incomes as they should. For the sake of simplicity, in the pursue we omit the analysis of axiom 2 violations, as it is quite analogous to the one described for axiom 1 .

\section{BASIC DECOMPOSITIONS}

Following Podder [8], [9] and Podder and Chatterjee [10], $C_{T \mid X}$ can be decomposed as follows

$C_{T \mid X}=\frac{\mu_{V}}{\mu_{T}} r_{V \mid X} G_{V}-\frac{\mu_{V-S}}{\mu_{T}} r_{V-S \mid X} G_{V-S}-\frac{\mu_{C}}{\mu_{T}} r_{C \mid X} G_{C}$,

where $r_{Z \mid X}=C_{Z \mid X} / G_{Z}, Z=V,(V-S), C$.

Note that $-1 \leq r_{Z \mid X} \leq 1$, as $-G_{Z} \leq C_{Z \mid X} \leq G_{Z}$.

By making use of (3), the index $R S$ can be represented as follows

$$
\begin{aligned}
R S & =\frac{\mu_{T}}{\mu_{Y}}\left[\frac{\mu_{V}}{\mu_{T}}\left(r_{V \mid X} G_{V}-G_{X}\right)\right] \\
& +\frac{\mu_{T}}{\mu_{Y}}\left[-\frac{\mu_{V-S}}{\mu_{T}}\left(r_{V-S \mid X} G_{V-S}-G_{X}\right)-\frac{\mu_{C}}{\mu_{T}}\left(r_{C \mid X} G_{C}-G_{X}\right)\right]
\end{aligned}
$$

We can analogously decompose $G_{T}$

$G_{T}=\frac{\mu_{V}}{\mu_{T}} r_{V \mid T} G_{V}-\frac{\mu_{V-S}}{\mu_{T}} r_{V-S \mid T} G_{V-S}-\frac{\mu_{C}}{\mu_{T}} r_{C \mid T} G_{C}$.

For what concerns $G_{Y}$ and $C_{Y \mid X}$, we have

$G_{Y}=\frac{\mu_{X-V}}{\mu_{Y}} r_{X-V \mid Y} G_{X-V}-\frac{\mu_{V-S}}{\mu_{Y}} r_{V-S \mid Y} G_{V-S}-\frac{\mu_{C}}{\mu_{Y}} r_{C \mid Y} G_{C}$ 


$$
C_{Y \mid X}=\frac{\mu_{X-V}}{\mu_{Y}} r_{X-V \mid X} G_{X-V}-\frac{\mu_{V-S}}{\mu_{Y}} r_{V-S \mid X} G_{V-S}-\frac{\mu_{C}}{\mu_{Y}} r_{C \mid X} G_{C}, \text { (7) }
$$

respectively. On the basis of the decompositions (3)-(5), and (6)-(7), we can decompose the contribution to axiom violations due to the marginal tax-rates, deductions and tax credits.

\section{Decomposing AXIOM Violations}

The KL measure of axiom 1 violation, can be decomposed as $A V_{1}^{T}=\tau\left(G_{T}-C_{T \mid X}\right)=A A V_{1}^{V}+A A V_{1}^{D}+A A V_{1}^{C}$,

where

$$
A A V_{1}^{V}=\frac{\mu_{V}}{\mu_{V}}\left(r_{V \mid T}-r_{V \mid X}\right) G_{V}, A A V_{1}^{C}=-\frac{\mu_{C}}{\mu_{V}}\left(r_{C \mid T}-r_{C \mid X}\right) G_{C}
$$

and $A A V_{1}^{D}=-\frac{\mu_{V-S}}{\mu_{V}}\left(r_{V-S \mid T}-r_{V-S \mid X}\right) G_{V-S}$.

According to Pellegrino and Vernizzi [6], let's now write

$$
C_{Z \mid E}=\frac{1}{2 \mu_{Z} N^{2}} \sum_{i=1}^{K} \sum_{j=1}^{K}\left(z_{i}-z_{j}\right) p_{i} p_{j} I_{i-j}^{Z \mid E}, I_{i-j}^{Z \mid E}=\left\{\begin{array}{cc}
1: & e_{i}>e_{j} \\
-1: & e_{i}<e_{j} \\
I_{i-j}^{Z}: & e_{i}=e_{j}
\end{array}\right.
$$

$Z=V,(V-S), C ; \quad E=T, X$.

We can observe that, for what concerns $\left(z_{i}-z_{j}\right)$ the contribute to axiom violation

i) is zero whenever the sign of $\left(t_{i}-t_{j}\right)$ is the same as the sign

$$
\text { of }\left(x_{i}-x_{j}\right) \text {, independently from the sign of }\left(z_{i}-z_{j}\right) \text {; }
$$

ii) is positive whenever the sign of $\left(z_{i}-z_{j}\right)$ is the same as the sign of $\left(t_{i}-t_{j}\right)$, and opposite to the sign of $\left(x_{i}-x_{j}\right)$;

iii) is negative whenever the sign of $\left(z_{i}-z_{j}\right)$ is the same as the

sign of $\left(x_{i}-x_{j}\right)$, and opposite to the sign of $\left(t_{i}-t_{j}\right)$.

For what concerns the KL measure of axiom 3 violation, it can be decomposed as

$$
A V_{3}^{T}=\left(G_{Y}-C_{Y \mid X}\right)=A A V_{3}^{V}+A A V_{3}^{D}+A A V_{3}^{C},
$$

where

$$
\begin{aligned}
& A A V_{3}^{V}=\frac{\mu_{X-V}}{\mu_{Y}}\left(r_{X-V \mid Y}-r_{X-V \mid X}\right) G_{X-V}, \\
& A A V_{3}^{D}=\frac{\mu_{V-S}}{\mu_{Y}}\left(r_{V-S \mid Y}-r_{V-S \mid X}\right) G_{V-S} \\
& A A V_{3}^{C}=\frac{\mu_{C}}{\mu_{Y}}\left(r_{C \mid Y}-r_{C \mid X}\right) G_{C},
\end{aligned}
$$

Here similar considerations apply as those presented for $A V_{1}^{T}$.

\section{CONCLUSION}

The instruments presented in this paper can be applied to investigate how the combination of the marginal tax schedule, of deduction and tax credits fulfill the aim of equity, according to KL axioms. The paper links together the contributions of Pfäler [7]; Lambert [3] and Onrubia et Al. [5], with the axiomatic approach presented by Kakwani and Lambert [2]. Differently from Pfäler, Lambert and Onrubia et Al, we do not consider explicitly deductions, as we think that their effect can be better evaluated by inspecting the distribution of the tax revenue without deductions, versus the one obtained after their application.

\section{REFERENCES}

[1] P. Di Caro, "Redistribution in real-world PIT: Evidence from italian tax records,",Dipartimento delle Finanze, MEF, WP No. 2. Technical report, 2018.

[2] N. C. Kakwani and P. J. Lambert, "On measuring inequity in taxation: a new approach," in European Journal of Political Economy, vol.14, pp.369-380, 1998. https://doi.org/10.1016/S0176-2680(98)00012-3

[3] P. J. Lambert, The distribution and redistribution of income. Manchester University Press, 2001.

[4] E. Mazurek and A. Vernizzi, "Some considerations on measuring the progressive principle violations and the potential equity in income tax systems," in Statistics in Transition, vol.14, pp.467-486, 2013.

[5] J. Onrubia, F. Picos-Sanchez and M. del Carmen Rodado, "Rethinking the Pfähler-Lambert decomposition to analyse real-world personal income taxes," in International Tax and Public Finance, vol. 21, pp.796-812, 2014. https://doi.org/10.1007/s10797-014-9316-1

[6] S. Pellegrino and A. Vernizzi, "On measuring violations of the progressive principle in income tax systems," in Empirical Economics, vol. 45, pp.239-245, 2013. https://doi.org/10.1007/s00181-012-0613-1

[7] W. Pfähler, "Redistributive effect of income taxation: decomposing tax base and tax rates effects," in Bulletin of Economic Research, vol. 42, pp.121-129, 1990. https://doi.org/10.1111/j.1467-8586.1990.tb00295.x

[8] N. Podder, "The disaggregation of the Gini coefficient by factor components and its applications to Australia," in Review of Income and Wealth, vol. 39, pp.51-61, 1993. https://doi.org/10.1111/j.1475-4991.1993.tb00437.x

[9] N. Podder, "A new decomposition of the Gini coefficient among groups and its interpretations with applications to Australia," in Sankhya: The Indian Journal of Statistics, Series B, vol. 55, pp.262-271, 1993.

[10] N. Podder and S. Chatterjee, "Sharing the national cake in post reform New Zealand: income inequality trends in terms of income sources," in Journal of Public Economics, vol. 86, pp.1-27, 2002. https://doi.org/10.1016/S0047-2727(01)00117-7 